Enfoques. Revista de Investigación en Ciencias de la Administración http://doi.org/10.33996/revistaenfoques.v2i7.40 No. 7 | Volumen 2 | Julio - Septiembre 2018

\section{EFECTOS DE LA APLICACIÓN DE LA LEY ORGÁNICA DEL TRABAJO, LOS TRABAJADORES Y LAS TRABAJADORAS SOBRE EL CÁLCULO DE LA GARANTÍA DE PRESTACIONES SOCIALES}

\author{
EFFECTS OF THE APPLICATION OF THE ORGANIC LAW ON \\ LABOR, WORKERS AND WORKERS ON THE CALCULATION OF \\ THE GUARANTEE OF SOCIAL BENEFITS
}

\author{
Jesús Arciniegas, Leunam Montaner y Carlos Palencia
}

Artículo recibido 22 abril 2018 | Arbitrado mayo-junio 2018| Publicado 01 de julio 2018

\section{Resumen}

La investigación que se resume en este artículo tuvo como objetivo evaluar los efectos que ocasiona la aplicación de la Ley Orgánica del Trabajo, en cuanto a los distintos aspectos que componen el cálculo de las prestaciones sociales en el personal docente del departamento de Contabilidad de la Facultad de Ciencias Económicas y Sociales de la Universidad de Carabobo. La metodología implementada en esta investigación fue de naturaleza descriptiva y cuantitativa, apoyado en un trabajo de campo, con el uso de la entrevista como instrumento de recolección de información. El estudio concluyó que los beneficios que la nueva legislación laboral venezolana aporta a los trabajadores son más que todo individuales. Esta ley beneficia a la población que actualmente trabaja en el sector formal, público o privado respectivamente. En definitiva, el Estado debe tener una visión holística de la sociedad y en función de esta amplia visión tomar las decisiones apropiadas que beneficien a la mayor cantidad de sectores posibles que integran al país.

Palabras clave: Ley Orgánica del Trabajo; personal docente universitario; régimen de prestaciones sociales; estudio comparativo

\begin{abstract}
The research that is summarized in this article aimed to evaluate the effects caused by the application of the Organic Labor Law, in terms of the different aspects that make up the calculation of social benefits in the teaching staff of the Department of Accounting of the Faculty of Economic and Social Sciences of the University of Carabobo. The methodology implemented in this research was descriptive and quantitative in nature, supported by a field work, with the use of the interview as an information collection tool. The study concluded that the benefits that the new Venezuelan labor legislation brings to workers are mostly individual. This law benefits the population currently working in the formal, public or private sector respectively. In short, the State must have a holistic vision of society and, based on this broad vision, make appropriate decisions that benefit the greatest possible number of sectors that make up the country.
\end{abstract}

Key words: Organic Labor Law; university teaching staff; Social Benefits scheme; Comparative study

\section{Jesús Arciniegas}

oswaldoelparcee@hotmail.com Universidad de Carabobo, Venezuela

Licenciado en Administración Comercial egresado de la Universidad de Carabobo, Venezuela.

\section{Leunam Montaner}

leunam0844@hotmail.com

Universidad de Carabobo, Venezuela

Licenciado en Contaduría Pública egresado de la Universidad de Carabobo, Venezuela.

\section{Carlos Palencia}

leunam0844@hotmail.com

Universidad de Carabobo, Venezuela

Licenciado en Administración Comercial, Universidad de Carabobo, Venezuela. 


\section{INTRODUCCIÓN}

Los trabajadores, a lo largo de la historia, son quienes con su fuerza de trabajo han construido el mundo conocido hasta hoy, levantando pueblos que posteriormente fueron ciudades $y$ construyendo caminos que posteriormente fueron fundamentales vías de comunicación entre ciudades y países; es por ello que cuando en el estudio se refiere a los trabajadores, se habla de esa columna vertebral que sostiene a cada familia $y$, en consecuencia, a la sociedad.

Por otra parte, las leyes son instrumentos creados por el hombre para regular su conducta y que en teoría su cumplimiento ha de proporcionarle armonía a la sociedad. Como ya ha sido mencionado, los trabajadores son componente fundamental de la sociedad y de la humanidad, por lo tanto una ley que trate sobre las condiciones que deben cumplirse por parte del empleador y los trabajadores al momento de establecerse una relación laboral es un tema neurálgico al que debe prestársele la adecuada atención.

En Venezuela, desde principios del siglo $\mathrm{XX}$, sus gobernantes sintieron la necesidad de establecer un cuerpo de legislaciones para amparar al trabajador, que, para entonces, estaba en condiciones precarias desde el punto de vista laboral, es decir, no existía ley alguna que los protegieran. Es desde entonces que se inician las promulgaciones de leyes que empezaron a proteger al trabajador. A medida que pasó el tiempo dichas leyes se volvieron poco prácticas para el rápido desarrollo por el que atravesaba el país, y es por ello que se inicia el proceso de reformulación de las leyes desde 1936 hasta el presente.

Dentro de este orden de ideas, se ubica un tema fundamental dentro del marco legal que ampara a los trabajadores, las prestaciones sociales, las cuales son un beneficio de todo trabajador, este beneficio consiste en una suma de dinero que el empleador está obligado a cancelar a sus trabajadores de acuerdo a la antigüedad de los mismos, al salario y a las utilidades que la empresa genere. Según la Constitución venezolana "El salario y las prestaciones sociales son créditos laborales de exigibilidad inmediata. Toda mora en su pago genera intereses, los cuales constituyen deudas de valor y gozarán de los mismos privilegios y garantías de la deuda principal" (Artículo 92 de la Constitución de la República Bolivariana de Venezuela, 1999).

En Venezuela la garantía de prestación de antigüedad constituyen un tema muy importante para las organizaciones, tanto públicas como privadas, las cuales son establecidas y reguladas por las distintas leyes que benefician a los trabajadores en el desarrollo y al término de su relación laboral como son: la Constitución de la República Bolivariana de Venezuela, Ley Orgánica del Trabajo y Reglamento de la Ley Orgánica del Trabajo.

En este sentido, para el mes de mayo de 2012, se aprobó la Ley Orgánica del Trabajo, los Trabajadores y Trabajadoras (LOTTT) la cual viene a sustituir a la Ley Orgánica del Trabajo (LOT), vigente desde el año 1997. La aplicación de esta nueva ley implica cambios y por supuesto que la garantía de prestaciones sociales no escapa de las distintas novedades contenidas en la LOTTT.

Las prestaciones sociales no sólo son un paquete social al momento del despido o la terminación de la relación de trabajo, también recompensan la antigüedad en el servicio, además tiene un carácter familiar porque representa una reserva para el trabajador y su familia en sus necesidades estratégicas. 
Para la aprobación de la vigente Ley Orgánica del Trabajo, los Trabajadores y Trabajadoras, el gobierno nacional, quien promovió la creación de la misma, afirmó reiteradamente por distintos medios de comunicación que se hizo una consulta nacional de trabajadores de los distintos sectores que componen económicos a nivel nacional, todo esto con la finalidad de promover la justicia social, mejores condiciones laborales, en fin, nuevas prerrogativas para el sector laboral.

Por lo antes expuesto y por lo que reza el artículo de la Constitución nacional previamente citado, surgió la siguiente interrogante: ¿Cuáles efectos ocasiona la aplicación de la nueva Ley Orgánica del Trabajo los Trabajadores y Trabajadoras (LOTTT) sobre los distintos aspectos que componen la prestación de antigüedad en el personal docente del Departamento de Contabilidad de la Facultad de Ciencias Económicas y Sociales de la Universidad de Carabobo?

Aunado a ello, el estudio se planteó como objetivo la evaluación de los efectos que ocasiona la aplicación de la Ley Orgánica del Trabajo, los Trabajadores y las Trabajadoras en cuanto a los distintos aspectos que componen la prestación de antigüedad en el personal docente del Departamento de Contabilidad de la Facultad de Ciencias Económicas y Sociales de la Universidad de Carabobo.

Para el logro del propósito del presente estudio, se valoraron las visiones de distintos teóricos que problematizan sobre la temática que se investigó. Al respecto, según Urqujo y Bonilla (2004) la remuneración del Trabajo se entiende como al total de pago que recibe un trabajador por la prestación de su servicio, lo que lleva a que algunos autores consideren la remuneración como una contraprestación. El diccionario de la Real Academia Española define la palabra remuneración como "la acción o efecto de remunerar", o lo que se da o sirve para remunerar. La forma de remuneración más usual se conoce como salario o sueldo. Desde el punto de vista económico se entiende como salario, a la remuneración cierta, anticipada a la producción, periódicamente recibida, establecida sobre una base contractual, como debida, por el trabajo independiente.

En Venezuela la Ley Orgánica del Trabajo (LOT) lo define como la remuneración, provecho o ventaja, cualquiera fuese su denominación o método de cálculo, siempre que pueda evaluarse en efectivo, que corresponda al trabajador por la prestación de sus servicios. El salario es un concepto fundamental para efectos de esta investigación ya que es el elemento básico empleado en el sistema de prestaciones sociales.

Por otro lado, las prestaciones sociales, son beneficios adquiridos por el trabajador, los cuales le compensan su tiempo en la empresa, y le protegen en caso de despido injustificado, justificado o al cesar la relación de trabajo. Cabe agregar, que las prestaciones sociales causadas que se cancelarán y abonarán mensualmente, bien en un fideicomiso, en un fondo de prestaciones o en la contabilidad de la empresa, a favor de cada trabajador y atendiendo a la voluntad de este. El trabajador podrá disponer del monto total de dichos abonos al término de la relación laboral, sin prejuicio de que la reforma de la Ley Orgánica del Trabajo sancione los supuestos en los cuales los recursos de depósitos en dicho fondo podrán disponerse - avalar créditos solicitados por los trabajadores. 
Es evidente, entonces, que las prestaciones sociales persiguen esencialmente fines sociales de carácter familiar, ya que contribuyen una reserva para la familia de los que ganan un sueldo o salario, y representan en cierto modo para el trabajador un nivel socio-económico porque de una u otra forma le garantiza, tanto a él como a su familia, subsistir por un periodo determinado en el cual se encuentra sin empleo.

En Venezuela se entiende por prestaciones sociales la indemnización que debe cancelársele a un trabajador por sus años de servicio al término de la relación laboral. Hasta el 19 de Junio de 1997 el cálculo de las prestaciones sociales establecido en la Ley Orgánica del Trabajo se realizaba en base al último salario devengado por el trabajador, es decir, siguiendo la siguiente fórmula; número de años multiplicado por 30 días multiplicado por salario integral diario.

A lo largo de los años la legislación de Venezuela ha implantado varios cambios en procura de la protección de los derechos de los laborales. Hoy en día se rige por la Ley Orgánica del Trabajo, los Trabajadores y las Trabajadoras (2012), la cual implica un avance significativo en los cálculos de las prestaciones sociales y otros beneficios.

Entre los cambios más significativos de la reforma de la Ley, se encuentra la nueva definición de salario y la modificación para el cálculo de las prestaciones sociales y sus intereses, ahora llamada "Prestación de Antigüedad", la indemnización por despido injustificado, y para los trabajadores que pasan del régimen anterior al nuevo, la compensación por transferencia.

En este mismo orden de ideas, es oportuno retomar lo expuesto por Rugeles y Carrillo (2007) cuando señalan que, el Fideicomiso constituye una de las figuras jurídicas más versátiles, puesto que cuenta con un campo de operaciones muy diverso, posee características propias, y es de gran flexibilidad. A la realización de un contrato fiduciario, existen diversas modalidades que se adaptan a las exigencias de los clientes, de forma tal que cubran fines diferentes de manera efectiva; dentro de los cuales se encuentran: los de administración, infraestructura, educación, garantía, inversión, laboral entre otros.

\section{MATERIALES Y MÉTODOS}

La investigación que se resume en este artículo, se corresponde con un diseño no experimental, (Arial 2006) se considera por tanto, que esta investigación es no experimental debido a que se evaluaron los efectos que ocasiona la aplicación de la Ley Orgánica de los Trabajadores y las Trabajadoras en cuanto a los distintos aspectos que componen el cálculo de las prestaciones sociales en el personal docente del departamento de Contabilidad de la Facultad de Ciencias Económicas y Sociales de la Universidad de Carabobo, sin hacer ningún tipo de prueba sobre el fenómeno estudiado. El estudio tiene las características de una investigación de campo y se apoya en una investigación documental.

El estudio estuvo apoyado en la modalidad de investigación documental, ya que se realizaron las revisiones bibliográficas y análisis de documentos legales pertinentes para elaborar un estudio comparativo de la Ley Orgánica del Trabajo (1997) y la Ley Orgánica del Trabajo, los Trabajadores y las Trabajadoras (2012) en cuanto a los distintos aspectos que componen el cálculo de las prestaciones sociales. Se utilizó el nivel descriptivo, ya que se caracterizaron los elementos asociados con el cálculo de las 
prestaciones sociales de los profesores del Departamento de Contabilidad de la Facultad de Ciencias Económicas y Sociales de la Universidad de Carabobo.

La población referida a esta investigación estuvo compuesta por cuarenta y nueve personas, que son los docentes que conforman el Departamento de Contabilidad de la Facultad de Ciencias Económicas y Sociales de la Universidad de Carabobo, quienes pueden aportar mediante sus percepciones y experiencia información relevante para los resultados de la investigación que se va a llevar a cabo. La muestra la conforma cinco informantes claves, docentes que del Departamento de Contabilidad de la Facultad de Ciencias Económicas y Sociales de la Universidad de Carabobo.

Para esta investigación, las técnicas de recolección de datos utilizadas fueron el análisis documental y la entrevista. Se utilizó para hacer el estudio comparativo la Ley Orgánica del Trabajo (1997) y la Ley Orgánica del trabajo, los Trabajadores y las Trabajadoras (2012) en cuanto a los distintos aspectos que componen el cálculo de las prestaciones sociales.

\section{RESULTADOS Y DISCUSIÓN}

Los resultados de la aplicación de los instrumentos de recolección de datos acordes según la metodología de la investigación, permitieron obtener información para:

1. Hacer un estudio comparativo de la Ley Orgánica del Trabajo (1997) y la Ley Orgánica del Trabajo, los Trabajadores y las Trabajadoras (2012) en cuanto a los distintos aspectos que componen el cálculo de las prestaciones sociales.
2. Determinar la variación porcentual del monto de prestaciones sociales del personal docente del departamento de contabilidad de la Facultad de Ciencias Económicas y Sociales de la Universidad de Carabobo al aplicar lo establecido en la ley orgánica del trabajo, los trabajadores y trabajadoras.

3. Evaluar las consecuencias que ocasiona la aplicación de la Ley Orgánica del Trabajo, los Trabajadores y las Trabajadoras en cuanto a los distintos aspectos que componen el cálculo de las prestaciones sociales en la unidad de estudio.

Objetivo 1. Elaborar un estudio comparativo de la Ley Orgánica del Trabajo (1997) y la Ley Orgánica del Trabajo, los Trabajadores y las Trabajadoras (2012) en cuanto a los distintos aspectos que componen el cálculo de las prestaciones sociales.

\section{Análisis del régimen de prestaciones sociales contemplado en la Ley Orgánica del Trabajo (1997).}

En el año 1997 se reforma parcialmente la LOT y se produce la modificación más sustantiva que ha sufrido la institución de las "prestaciones sociales". Dos artículos de la LOT (1997) resultan claves para entender el "nuevo" régimen de prestaciones sociales: el artículo 108 y el artículo 672. El primero, el artículo 108 , conjuntamente con los artículos 125,133 y 146 , desarrolla extensamente la institución laboral. El segundo, el 672, preserva los regímenes de prestaciones sociales de fuentes distintas a la Ley Orgánica del Trabajo. En este sentido, el régimen establecido en el artículo 108 tiene, entre otros, los caracteres siguientes: 
a) El derecho del trabajador a cobrar la "prestación de antigüedad" nace a partir del tercer mes de servicio ininterrumpido.

b) La liquidación es mensual, a razón de 5 días de salario del mes anterior por cada mes de servicio, con uno adicional de 2 días, a partir del segundo año de servicio, acumulativos hasta 30 días; en total, el trabajador pudiera alcanzar hasta 90 días de salario por concepto de "prestación de antigüedad" una vez alcanzados los 16 años de servicio.

c) A los fines de la colocación o depósito por parte del empleador de la "prestación de antigüedad", en la forma como ésta se causa, impera la voluntad del trabajador.

d) Lo depositado o acreditado mensualmente se pagará al término de la relación de trabajo y devengará intereses según las siguientes opciones: a) Al rendimiento del fideicomiso o los fondos de prestaciones de antigüedad o la tasa de mercado si fuere en una entidad financiera; b) A la tasa activa determinada por el BCV, si el trabajador hubiese requerido del empleador el lugar del depósito y éste no cumpliere lo solicitado; y, c). A la tasa promedio entre la activa y pasiva, determinada por el $B C V$, tomando como referencia los seis principales bancos comerciales $y$ universales del país, si fuere en la contabilidad de la empresa.

e) El trabajador podrá retirar hasta el $75 \%$ de lo depositado por los motivos fijados por la Ley.

f) Los intereses serán acreditados mensualmente y pagados al cumplir cada año de servicio, salvo que el trabajador, mediante manifestación escrita, decidiere capitalizarlos.
El campo de aplicación subjetivo de este régimen comprende tanto a los trabajadores subordinados del sector público como del sector privado. Por otra parte, el artículo 672 de la LOT regula los regímenes de prestaciones sociales que no transitaron $\mathrm{o}$ migraron al nuevo régimen (108 LOT) sino que permanecieron bajo las condiciones anteriores. Este artículo es muy claro y preciso cuando establece que: "Los regímenes de fuentes distintas a esta ley, que en su conjunto fueren más favorables al sancionado en los artículos 108, 125, 133 y 146 de esta ley, se aplicarán con preferencia en su integridad y no serán acumulativos en ningún caso". No hay duda alguna que el legislador orgánico se refiere a los regímenes convencionales, es decir, los alcanzados por los trabajadores mediante la contratación colectiva u otro tipo de convención laboral.

Otros artículos de la LOT que fundamentan y dan protección a la institución de las "prestaciones sociales" son el artículo 159 y 160 referidos al privilegio del salario, las prestaciones e indemnizaciones y cualesquiera otros créditos debidos al trabajador con ocasión de la relación de trabajo.

\section{Análisis del régimen de prestaciones sociales contemplado en la Ley Orgánica del Trabajo, los Trabajadores y las Trabajadoras (2012)}

La Ley Orgánica del Trabajo de (2012) establece un nuevo régimen de Prestaciones Sociales que contempla la recuperación de la "retroactividad", mediante el cual, al trabajador culminar la relación laboral se le calcularán sus prestaciones con el último salario y tomando en cuenta los años de servicio. Además, el trabajador tendrá prestaciones sociales desde el primer día de 
trabajo y no a partir de los tres meses como establecía la Ley derogada. Los depósitos de prestaciones sociales se harán donde el trabajador decida, en la contabilidad de la empresa, en un fideicomiso o en el Fondo Nacional de Prestaciones Sociales.

Como se señala en el Artículo 142 de la Ley Orgánica del Trabajo vigente referente a Garantía y cálculo de prestaciones sociales: las prestaciones sociales se protegerán, calcularán y pagarán de la siguiente manera:

a) El patrono o patrona depositará a cada trabajador o trabajadora por concepto de garantía de las prestaciones sociales el equivalente a quince días cada trimestre, calculado con base al último salario devengado.

b) Adicionalmente y después del primer año de servicio, el patrono o patrona depositará a cada trabajador o trabajadora dos días de salario, por cada año, acumulativos hasta treinta días de salario.

c) Cuando la relación de trabajo termine por cualquier causa se calcularán las prestaciones sociales con base a treinta días por cada año de servicio o fracción superior a los seis meses calculada al último salario.

d) El trabajador o trabajadora recibirá por concepto de prestaciones sociales el monto que resulte mayor entre el total de la garantía depositada de acuerdo a lo establecido en los literales $a$ y $b$, y el cálculo efectuado al final de la relación laboral de acuerdo al literal c.

e) Si la relación de trabajo termina antes de los tres primeros meses, el pago que le corresponde al trabajador o trabajadora por concepto de prestaciones sociales será de cinco días de salario por mes trabajado o fracción.

f) El pago de las prestaciones sociales se hará dentro de los cinco días siguientes a la terminación de la relación laboral, si no el pago generará intereses de mora a la tasa activa determinada por el Banco Central de Venezuela.

\section{Alcance de la LOTTT en las Prestaciones Sociales de los trabajadores $y$ trabajadoras venezolanos}

Las leyes laborales siempre se han caracterizado por ser correctoras en el ámbito social y por producir consecuencias de orden económico, pues establecen normas especiales relacionadas con la seguridad social, el salario, el reconocimiento del tiempo de servicio del trabajador, entre otras. Con la nueva Ley Orgánica del Trabajo (LOTTT), todavía es difícil definir los alcances de sus implicaciones; pese a lo extenso de la Ley, no existe una visión clara de cómo va a funcionar el mercado laboral; siendo un ejemplo de ello, el artículo 10 de la normativa, que da amplias potestades al Gobierno Nacional de regir en materia laboral a través de decretos y reglamentos, en una especie de habilitante en materia laboral.

Por tanto, aún existe confusión en la población, debido a que no se conocen realmente los alcances de la Ley; en el cual, se desglosarán alrededor de once leyes especiales, aparte del reglamento que estará rigiendo, por lo que hay que destacar la importancia de estudiarla detalladamente para conocer sus implicaciones a posteriores. 
Cuadro 1 - Comparación de la Ley Orgánica del Trabajo (1997) y la Ley Orgánica del Trabajo, los trabajadores y trabajadoras (2012) en cuanto a los aspectos que comprenden el cálculo de prestaciones sociales.

\begin{tabular}{|c|c|c|}
\hline Aspecto & LOT & LOTTT \\
\hline $\begin{array}{l}\text { Nombre del componente } \\
\text { principal de las prestaciones } \\
\text { sociales. }\end{array}$ & Prestación de antigüedad. & $\begin{array}{l}\text { Garantía de prestaciones } \\
\text { sociales. }\end{array}$ \\
\hline $\begin{array}{l}\text { Frecuencia del abono de } \\
\text { prestación de antigüedad o } \\
\text { garantía de prestaciones } \\
\text { sociales. }\end{array}$ & Mensual. & Trimestral. \\
\hline $\begin{array}{l}\text { Momento de nacimiento del } \\
\text { derecho a prestaciones } \\
\text { sociales. }\end{array}$ & $\begin{array}{l}\text { A partir del tercer mes } \\
\text { ininterrumpido de la relación } \\
\text { laboral. }\end{array}$ & $\begin{array}{l}\text { Desde el primer día de la } \\
\text { relación laboral. }\end{array}$ \\
\hline $\begin{array}{l}\text { Salario para efectos del cálculo } \\
\text { de prestaciones sociales. }\end{array}$ & Salario integral. & Salario integral. \\
\hline $\begin{array}{l}\text { Cantidad de días por concepto } \\
\text { de abono anual. }\end{array}$ & $\begin{array}{l}2 \text { días acumulativos hasta } 30 \\
\text { días que deben ser cancelados } \\
\text { al trabajador. }\end{array}$ & $\begin{array}{l}2 \text { días acumulativos hasta } \\
30 \text { días que pueden ser } \\
\text { abonados a la garantía de } \\
\text { prestaciones sociales o } \\
\text { canceladas al trabajador. }\end{array}$ \\
\hline
\end{tabular}

Objetivo 2. Determinar los montos que corresponden en cuanto a prestación de antigüedad del personal docente del departamento de contabilidad de la Facultad de Ciencias Económicas y Sociales de la Universidad de Carabobo al aplicar lo establecido en la Ley Orgánica del Trabajo (1997) y lo establecido en la Ley Orgánica del Trabajo, los Trabajadores y Trabajadoras (2012).

Para evaluar los beneficios del nuevo régimen de prestaciones desde el punto de vista monetario, se procedió a entrevistar a una muestra de cinco (5) profesores del Departamento de Contabilidad de la Facultad de Ciencias Económicas y Sociales de la Universidad de Carabobo, a quienes se les solicitó información respecto a: año de ingreso como docente contratado por credenciales de la Universidad de Carabobo; escalafón y a qué fecha ascendió; salario básico; remuneraciones conforman su salario integral y último pago por concepto de aguinaldo y bono vacacional.

Luego se procedió a hacer el cálculo de los montos que correspondiente, tomando en cuenta las siguientes premisas para realizar el cálculo:

a) Los elementos que conforman el cálculo de las prestaciones son los contemplados en la I Convención Colectiva Única de Trabajadores del Sector Universitario 2015-2016.

b) Se prevé que cada año habrá un aumento del $10 \%$ del salario en ambos casos (LOT y LOTTT).

c) Al cuarto año habrá un aumento del 10 $\%$ en todas las primas.

d) Para efectos del cálculo de intereses se aplicó una tasa del $17 \%$ anual todo los años. 
Objetivo 3. Evaluar las consecuencias que ocasiona la aplicación de la Ley Orgánica del Trabajo, los Trabajadores y las Trabajadoras en cuanto a los distintos aspectos que componen el cálculo de la garantía de prestaciones sociales en la unidad de estudio.

En la actualidad, las leyes en el país están encaminadas a un proceso de cambios, contemplando la gestión normativa de manera innovadora para las futuras generaciones como actividad prioritaria y una estrategia global no sólo en el sentido de coadyuvar al mejor cumplimiento de los fines que el legislador tiene, sino con la finalidad de conseguir ventajas que favorezcan tanto a los trabajadores como a los patronos.

Es por ello necesario aplicar por parte de quien está interpretando la norma, estrategias laborales en el proceso para el cálculo de las prestaciones sociales de los trabajadores, el cual sea de fácil comprensión y funcional. Este conocimiento permite al patrono el poder evaluar y utilizar las herramientas o recursos efectivos $y$ eficaces para hacer cumplir las necesidades requeridas por los trabajadores a su cargo y les sea garantizado de forma segura el pago de sus prestaciones sociales de acuerdo a la Ley del Trabajo.

Bajo esta perspectiva, el cambio que ha tenido la ahora vigente Ley Orgánica del Trabajo, los Trabajadores y las Trabajadoras, ha creado nuevos escenarios, ratificando el papel que juegan las prestaciones sociales, para poder ofrecerles a cada uno de ellos una garantía y cálculo conforme a la norma; donde el patrono, debe estar capacitado en el conocimiento de las características de los entornos que esta nueva ley señala, de otra manera no se pueden colocar en la perspectiva de garantizar la ayuda o andamiaje que ofrece la LOTTT.

\section{CONCLUSIONES}

Las propuestas en materias de prestaciones sociales han pretendido acabar con la incertidumbre sobre la indemnización a recibir que existe por parte de los trabajadores al momento de la finalización de la relación de trabajo y a dar solidez a la capacidad de ahorro a mediano plazo. En definitiva, y por el panorama, ha existido y urge la modificación del sistema de prestaciones sociales, así como la necesidad de presentar un conjunto de alternativas que permitan al trabajador seleccionar la más adecuada a su necesidad.

Basado en esto, la investigación referida tuvo como propósito evaluar los efectos (principalmente económicos) que ocasiona la aplicación de la Ley Orgánica del Trabajo, los Trabajadores y las Trabajadoras en cuanto a los distintos aspectos que componen el cálculo de las prestaciones sociales en el personal docente del Departamento de Contabilidad de la Facultad de Ciencias Económicas y Sociales de la Universidad de Carabobo. Al respecto, se elaboraron las siguientes conclusiones:

Con respecto a la comparación de la Ley Orgánica del Trabajo (1997) y la Ley Orgánica del Trabajo, los Trabajadores y las Trabajadoras (2012) de los distintos aspectos que componen el cálculo de las prestaciones sociales, se concluye que es difícil aún definir los alcancen de las aplicaciones de la nueva Ley en lo que se refiere a las prestaciones sociales de los trabajadores; primeramente por lo extenso de la ley misma y por otra parte porque no existe una visión clara de cómo va a funcionar el mercado laboral, ya que por los momentos no existe un reglamento de la nueva ley. 
De allí que se hizo fundamental para los autores de la investigación hacer un análisis profundo de la legislación vigente con los involucrados laborales, para así darle sentido a los nuevos papeles y funciones a quienes les corresponde aplicarla e ir progresivamente determinando las evaluaciones y análisis de sus alcances e impactos. Tempranamente empezaron los ajustes, como ha ocurrido entre el texto que se envió al Tribunal Supremo de Justicia y que una semana más tarde fue publicado en Gaceta Oficial relativa al pago de prestaciones sociales.

Asimismo, el nuevo sistema de prestaciones sociales establecido en la legislación vigente, para el fortalecimiento de los derechos laborales y la seguridad social, va más allá y excede de una simple reforma requerida y necesaria por una disposición transitoria al régimen de prestaciones sociales; porque incorpora normas que adaptan a la nueva ley laboral a la Constitución de la República Bolivariana de Venezuela.

Además, la Ley introduce importantes elementos de garantía y protección de los derechos laborales, como la recuperación de la retroactividad en las prestaciones, el mantener los fideicomisos; ratificándose así mismo, que las prestaciones sociales son créditos laborales de exigibilidad inmediata y que toda mora en su pago genera intereses, para lo cual se crea un Fondo de Prestaciones Sociales. Mediante la reforma de la Ley Orgánica del Trabajo, se crea un nuevo régimen para el derecho a prestaciones sociales consagrado en la constitución, el cual integra el pago de este derecho de forma proporcional al tiempo de servicio y calculado de conformidad con el último salario devengado, estableciendo un lapso para su prescripción de diez años.

Finalmente, con respecto a las consecuencias que ocasiona la aplicación de la Ley Orgánica del Trabajo, los Trabajadores y las Trabajadoras en cuanto a los distintos aspectos que componen el cálculo de las prestaciones sociales en la unidad de estudio, también se concluye que los beneficios que la nueva legislación laboral venezolana aporta a los trabajadores son más que todo individuales.

De allí, la relevancia del estudio para los investigadores; ya que permite a otros personas que tienen interés con el fenómeno y por tanto fijen posición al respecto, como las comunidades, las organizaciones no gubernamentales y sectores académico que puedan profundizar con su participación para futuros análisis sobre bases firmes del proceso de reforma laboral y tomen en consideración si los criterios de los organismos técnicos como la OIT, son mencionados y considerados en su contenido; esto da lugar a la llamada reforma puntual, que refiere a pocos temas pero de muy importante impacto en las relaciones laborales.

\section{REFERENCIAS}

Arias, F. (2006). El proyecto de Investigación, Guía para su elaboración. Tercera Edición. Caracas: Episteme

Constitución de la República Bolivariana de Venezuela (1999). Gaceta Oficial $\mathrm{N}^{\circ}$ 36860. Diciembre, 1999

Ley Orgánica del Trabajo (1997). Gaceta Oficial N $N^{\circ} 5152$ (Extraordinaria). Junio, 1997

LOT (2012) Ley Orgánica del Trabajo, los Trabajadores y Trabajadoras. Venezuela Gaceta Oficial N 5929

Rugeles, C. y Carrillo, M. (2007). Derecho Laboral Venezolano. Ediciones de la UCAB, Caracas

Urqujo, J. y Bonilla, J. (2004). La remuneración del trabajo. Manual para La gestión de sueldos y salarios. IIES. UCAB. Caracas 